\title{
Multiple Duodenal Lipomas as a Rare Cause of Upper Gastrointestinal Obstruction: Case Report and Literature Review
}

\author{
Maowei Peia ${ }^{\mathrm{a}}$, Mingrong $\mathrm{Hu}^{\mathrm{a}}$, b, Wenbin Chen ${ }^{\mathrm{a}}$, Chao Qin ${ }^{\mathrm{a}}$
}

\begin{abstract}
Duodenal lipomas are rare benign tumors and pose a diagnostic challenge as their symptoms are non-specific. In this article, we reported a case of duodenal lipoma presenting as upper gastrointestinal obstruction and reviewed the literature on relevant clinical manifestation, diagnosis and treatment. Our review of literature indicated that multiple duodenal lipomas as a cause of upper gastrointestinal obstruction as reported here are extremely rare. The preoperative computed tomography and magnetic resonance imaging are the key to diagnosis, and surgical resection is the most effective means for the management of such duodenal lipomas.
\end{abstract}

Keywords: Duodenal neoplasm; Lipoma; Lipomatosis; Obstruction; Duodenectomy

\section{Introduction}

Gastrointestinal lipomas are very rare with limited case reports present in literature. Recently, with the advances in endoscopy and imaging techniques such as computed tomography (CT) and magnetic resonance imaging (MRI), more cases have been diagnosed and treated. Herein, we reported our experience in the treatment of a 67-year-old woman who presented with gastrointestinal obstruction due to multiple duodenal lipomas.

\section{Case Report}

A 67-year-old woman (56 kg with a body mass index of 21.1 $\mathrm{kg} / \mathrm{m}^{2}$ ) presented with 1-day history of abdominal pain, vomit and constipation in the local clinic. CT of the abdomen showed

\section{Manuscript accepted for publication March 20, 2017}

aDepartment of General Surgery, Hangzhou Normal University Affiliated Hospital, Hangzhou, China

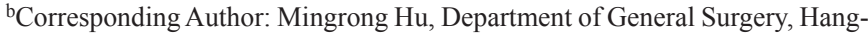
zhou Normal University Affiliated Hospital, No. 126, Wenzhou Road, Gongshu District, Hangzhou, Zhejiang, China. Email: hmr2012@163.com

doi: https://doi.org/10.14740/gr817w features of upper gastrointestinal obstruction with a suspicion of intussusception of a duodenum segment. The patient was transferred to our hospital for further treatment on October 16, 2016. She had a 5-year history of epigastric fullness and intermittent upper abdominal pain. She denied the history of hematemesis, melena, change in bowel habit or significant recent weight change. She did not have surgical history in the past. She denied family history of neoplasia. She was a nonsmoker and non-alcoholic drinker. On admission, she showed improvement in her symptoms and there were no significant findings except epigastric tenderness on physical examination. Hematological and biochemical parameters were normal except for $\mathrm{Hb}: 95 \mathrm{~g} / \mathrm{L}$ (normal range: 110 - $150 \mathrm{~g} / \mathrm{L}$ ), neutrophil ratios: $90.4 \%$ (normal range: $50-70 \%$ ), and C-reactive protein: $7.89 \mathrm{mg} / \mathrm{L}$ (normal range: $<5 \mathrm{mg} / \mathrm{L}$ ). Plain radiography of the abdomen showed bowel dilatation with air-fluid levels. CT of the abdomen revealed hypodense lesions with CT value of -64 Housefield Units (HU) to -138 HU located from the descending part of the duodenum to the proximal jejunum, which was suggestive of lipomas (Fig. 1). She was initially managed with fluid replacement and nasogastric aspiration and subsequently underwent MRI of the abdomen, which showed fat-containing mass lesions from the descending part of the duodenum to the proximal jejunum. The lesions were high-intensity on T1-weighted (Fig. 2a) and intermediately intense on T2weighted images, with a drop in signal on T1 and T2-weighted fat-suppressed images (Fig. 2b), consistent with a diagnosis of duodenal lipomas. As the obstruction did not fully resolve, we carried out an exploratory laparotomy under general anesthesia. On entering the peritoneal cavity, there was no obvious

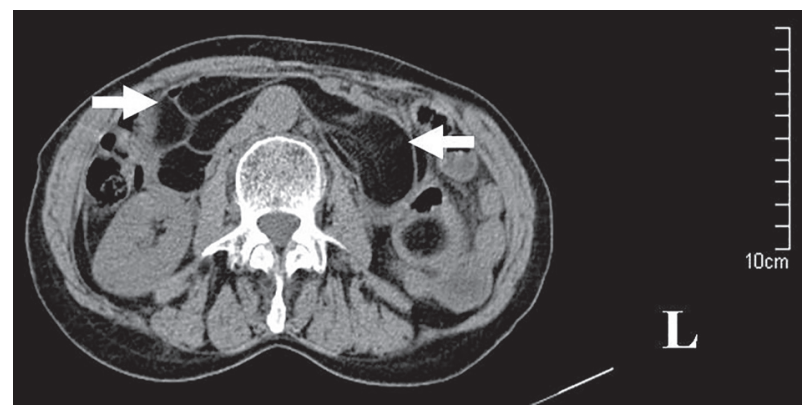

Figure 1. CT scan shows hypodense lesions located from the descending part of the duodenum to the proximal jejunum, suggestive of lipomas. 

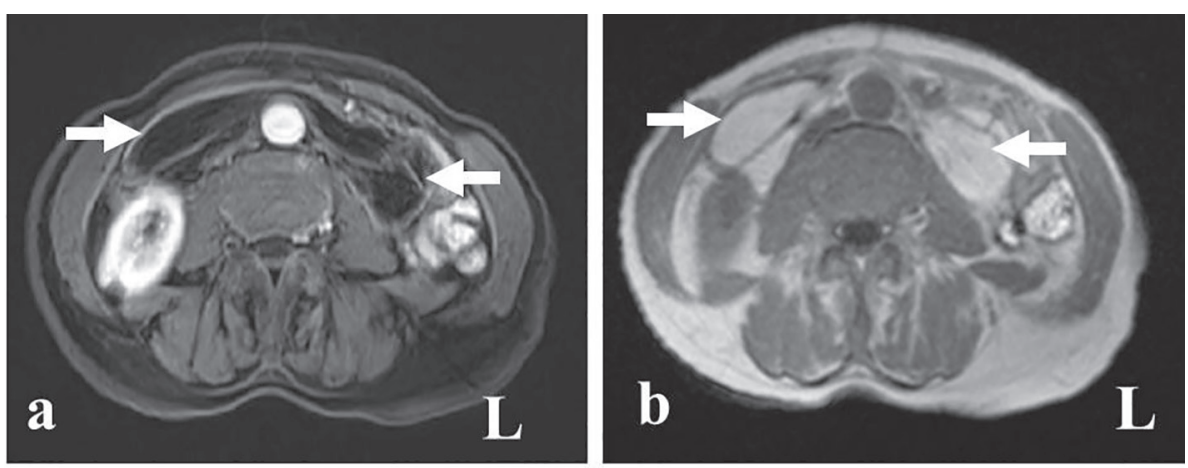

Figure 2. Panel (a) shows the lesions drop in signal on T1-weighted fat-suppressed image; panel (b) shows a well-demarcated, hyperintense mass on T1-weighted image (arrow).

intussusception of the duodenum. Intraoperatively, multiple, giant lipomas could be palpated in second, third and fourth part of duodenum and proximal jejunum and the rest of the bowel appeared normal. Duodenum $4 \mathrm{~cm}$ distal to ampulla was excised along with $14 \mathrm{~cm}$ of proximal jejunum (Fig. 3a). Intestinal continuity was maintained by end-to-end duodenojejunal hand-sewn anastomosis. Gastrostomy, jejunostomy and T tube in the common duct were performed in the meanwhile to prevent the anastomotic leakage. The patient was discharged from the hospital with successful recovery. The macroscopic appearance of the lesion is shown in Figure 3a. Final histopathological analysis confirmed the diagnosis of multiple, submucosal lipomas with sizes varying from 1.3 to $11.0 \mathrm{~cm}$ in maximal dimensions.

\section{Discussion}

A PubMed search was performed using the terms "duodenal lipoma" and "obstruction", which generated 28 articles. Of these 28 articles, 13 case reports published in English from 1951 to 2016 were identified for further review. Of them seven cases were single lipoma, five cases were multiple lipomas and one case did not record the number of lipoma(s) (Table 1) [112]. To date, two types of duodenal lipomas are reported in literature: submucosal, which is more common $(91.7 \%)$ as seen in our case and subserosal (8.3\%). Median age of presentation is 56.5 years (range, $12-71$ years) with female to male ratio of 9:5. Average maximum diameter of the mass is $6.1 \mathrm{~cm}$ (range,
$2.5-11 \mathrm{~cm}$ ). The location of duodenal lipoma presenting as obstruction is mostly seen in the second part of the duodenum, and the most effective means for treatment is transduodenal resection.

Lipomas of the duodenum are uncommon, and the etiology remains unknown. Duodenal lipomas are usually asymptomatic $[13,14]$, and if symptomatic, the common findings are bleeding, abdominal pain or obstruction. Symptoms are related to the characteristic of the lipoma, such as size and location. Duodenal lipomas are mostly seen in the second part (85.7\%). Obstruction-causing duodenal lipomas are extremely rare and may present as obstruction such as intestinal obstruction [17], gastric outlet obstruction [8-10], intussusception [11, 12] and obstructive jaundice [8]. The first case was reported by Kirkland et al in 1951 [1]. The case with obstructive jaundice reported by McGrath et al is particularly unusual [11]. The diameter of the tumors that caused obstruction are all greater than $2 \mathrm{~cm}$, and eight cases $(88.9 \%)$ of those are greater than 4 $\mathrm{cm}$ according to our literature review.

Diagnosis can be established by radiological, endoscopic or operative means. Current imaging modalities, such as CT and MRI can provide an accurate diagnosis [5, 15]. Duodenal lipoma appears on CT as a smooth-margined mass with a low Hounsfield unit value (range, -60 to -120 ), corresponding with the density of fat [15]. In the present case, the lesions were noted on CT with a value of -64 to $-138 \mathrm{HU}$ which is typical of lipomas. As for MRI, lipomas show high-intensity on T1-weighted images and usually present intermediate intensity on T2-weighted images. The loss of signal intensity on
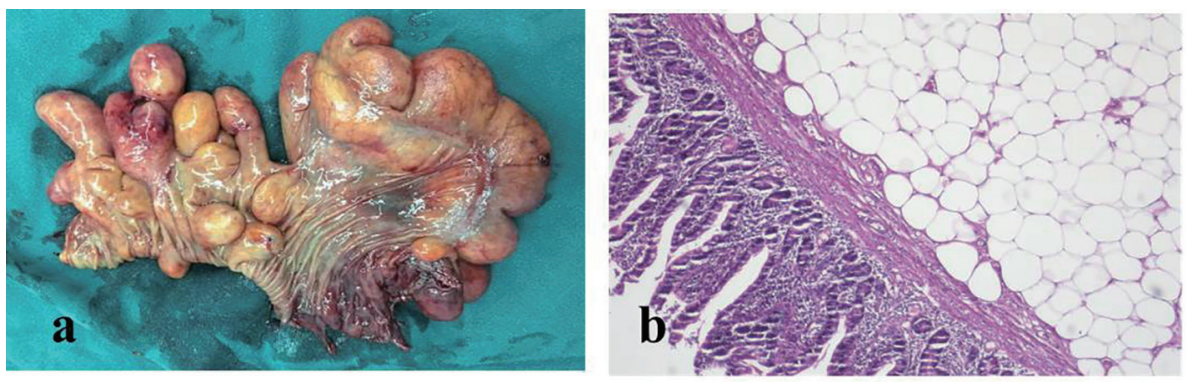

Figure 3. Panel (a) shows the macroscopic appearance of the duodenal lipoma with naked fat sign; panel (b) demonstrates pathological changes in the resected sample confirming the diagnosis of submucosa lipomas (H\&E, $\times 400)$. 


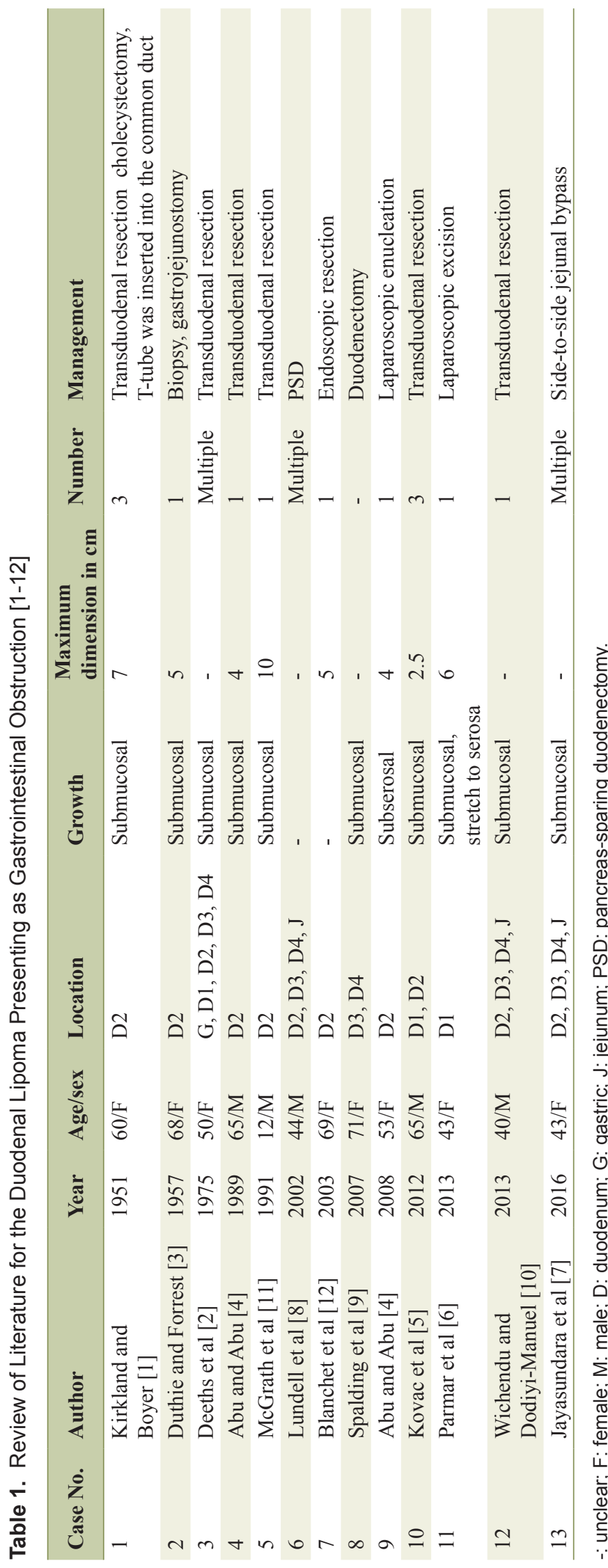

fat-suppressed images enables the final diagnosis by MRI [5]. Lipoma shows no contrast enhancement. Though CT and MRI are helpful in diagnosis but they are unable to precisely locate the origin of the lesions. Direct visualization at endoscopy provides the presence of submucosal protruding lesions, but further characterization is usually performed by endoscopic ultrasound (EUS). EUS is effective to provide information about the original layer, echogenicity, the depth and invasion. The typical EUS findings for duodenal lipomas are intense homogeneous hyperechoic lesions originating from the submucosa, with echo attenuation behind and/or inside the rear area [16].

Lipomas of the duodenum can be excised endoscopically or operatively. The pedunculated, small, solitary lipoma can be excised endoscopically [12]. Endoscopic management has been described in both the snaring technique [12] and the unroofing technique [17]. However, endoscopic management is not always feasible, and incomplete excision is also common in large lesions, which remains a reasonable concern, although there are no reports of recurrence of symptoms after incomplete excision. Operative management is indicated in cases in which endoscopy is not feasible, the nature of the lesion cannot be ascertained or if the clinical presentation such as intussusception requires surgery. It also has additional advantages, e.g., assuring complete excision of the lipoma, which is not always possible endoscopically. Operative management is mainly divided into four procedures, excision of the lipoma via a duodenotomy [1, 2, 5, 10, 11], a limited bowel resection [9], pancreas-sparing duodenectomy [8], or bypass [3, 7]. The type of procedure chosen to perform depends on the patient's conditions as well as the size and position of the lesion. In five cases, the surgery consisted of a reduction after duodenotomy and polypectomy. A segmental resection of the involved portion of the duodenum was performed in one case and the patient was fully recovered. Pancreas-sparing duodenectomy was performed in one case because the lipomas invaginated into the duodenojejunal flexure, and the patient had a history of obstructive jaundice. In case the excision of the entire duodenum is not safe, a bypass to the lesion is recommended. Laparoscopy has recently been described for the management of duodenal lipoma, which is a minimally invasive mode of surgical management with minimal postoperative pain and short hospitalization $[4,6]$. Our patient had rare multiple duodenal lipomas presenting with intestinal obstruction which was managed in time with duodenal resection and had uneventful recovery.

In conclusion, a case of multiple duodenal lipomas presenting as upper gastrointestinal obstruction, which is extremely rare, was diagnosed and successfully managed by surgical resection.

\section{References}

1. Kirkland WG, Boyer RA. Multiple lipomas of the duodenum: a case report. Gastroenterology. 1951;19(1):142147.

2. Deeths TM, Madden PN, Dodds WJ. Multiple lipomas of the stomach and duodenum. Am J Dig Dis. 1975;20(8):771-774.

3. Duthie HL, Forrest AP. Submucous lipoma of the duode- 
num. Br J Surg. 1957;45(190):201-202.

4. Abu Daff SN, Abu Daff NS. Laparoscopic enucleation of a duodenal lipoma, with review of the literature. Saudi Med J. 2008;29(3):455-457.

5. Kovac JD, Dunjic MK, Bjelovic M, Banko B, Lilic G, Milenkovic R, Micev M, et al. Magnetic resonance imaging features of multiple duodenal lipomas: a rare cause of intestinal obstruction. Jpn J Radiol. 2012;30(8):676-679.

6. Parmar AK, Bibyan M, Khandelwal R, Reddy PK. Laparoscopic management of a large duodenal lipoma presented as gastric outlet obstruction. JSLS. 2013;17(3):459462.

7. Jayasundara J, Sellahewa CS, Hall AD, Patel RT. A case of gastroduodenal lipomatosis. Ann R Coll Surg Engl. 2016;98(8):e203-e205.

8. Lundell L, Hyltander A, Liedman B. Pancreas-sparing duodenectomy: technique and indications. Eur J Surg. 2002;168(2):74-77.

9. Spalding DR, Isla AM, Thompson JN, Williamson RC. Pancreas-sparing distal duodenectomy for infrapapillary neoplasms. Ann R Coll Surg Engl. 2007;89(2):130-135.

10. Wichendu PN, Dodiyi-Manuel A. Gastric outlet obstruction from duodenal lipoma in an adult. Niger J Surg. 2013;19(2):79-81.

11. McGrath FP, Moote DJ, Langer JC, Orr W, Somers S. Duodenojejunal intussusception secondary to a duode- nal lipoma presenting in a young boy. Pediatr Radiol. 1991;21(8):590-591.

12. Blanchet MC, Arnal E, Paparel P, Grima F, Voiglio EJ, Caillot JL. Obstructive duodenal lipoma successfully treated by endoscopic polypectomy. Gastrointest Endosc. 2003;58(6):938-939.

13. Aydin HN, Bertin P, Singh K, Arregui M. Safe techniques for endoscopic resection of gastrointestinal lipomas. Surg Laparosc Endosc Percutan Tech. 2011;21(4):218-222.

14. Kadaba R, Bowers KA, Wijesuriya N, Preston SL, Bray GB, Kocher HM. An unusual cause of gastrointestinal bleeding: duodenal lipoma. Case Rep Gastroenterol. 2011;5(1):183-188.

15. Genchellac H, Demir MK, Ozdemir H, Unlu E, Temizoz O. Computed tomographic and magnetic resonance imaging findings of asymptomatic intra-abdominal gastrointestinal system lipomas. J Comput Assist Tomogr. 2008;32(6):841-847.

16. Chen HT, Xu GQ, Wang LJ, Chen YP, Li YM. Sonographic features of duodenal lipomas in eight clinicopathologically diagnosed patients. World J Gastroenterol. 2011;17(23):2855-2859.

17. Huang WH, Peng CY, Yu CJ, Chou JW, Feng CL. Endoloop-assisted unroofing for the treatment of symptomatic duodenal lipomas. Gastrointest Endosc. 2008;68(6):1234-1236. 